\title{
Large Time Scale Optimal Control of an Electrodynamic Tether Satellite
}

\author{
Robert Stevens* and William Wiesel ${ }^{\ddagger}$ \\ Air Force Institute of Technology, Wright-Patterson Air Force Base, Ohio 45433
}

DOI: $\underline{10.2514 / 1.34897}$

\begin{abstract}
Low-thrust propulsion systems offer a fuel-efficient means to maneuver satellites to new orbits; however, they can only perform such maneuvers when they are continuously operated for a long time. Such long-term maneuvers occur over many orbital revolutions, often rendering short time scale trajectory optimization methods ineffective. An approach to multirevolution large time scale optimal control of an electrodynamic tether is investigated for a tethered satellite system in low Earth orbit with atmospheric drag. Control is assumed to be periodic over several orbits because, under the assumptions of a nearly circular orbit, periodic control yields the only solution that significantly contributes to secular changes in the orbital parameters. The optimal control problem is constructed in such a way as to maneuver the satellite to a new orbit while minimizing a cost function subject to the constraints of the timeaveraged equations of motion by controlling current in the tether. Three optimal maneuvers were investigated for a $4 \mathrm{~km}$ tether in a $270 \mathrm{~km}$ initial orbit: maximum climb, maximum final inclination, and a minimum time orbit change. The resulting control solutions were propagated to verify their accuracy.
\end{abstract}

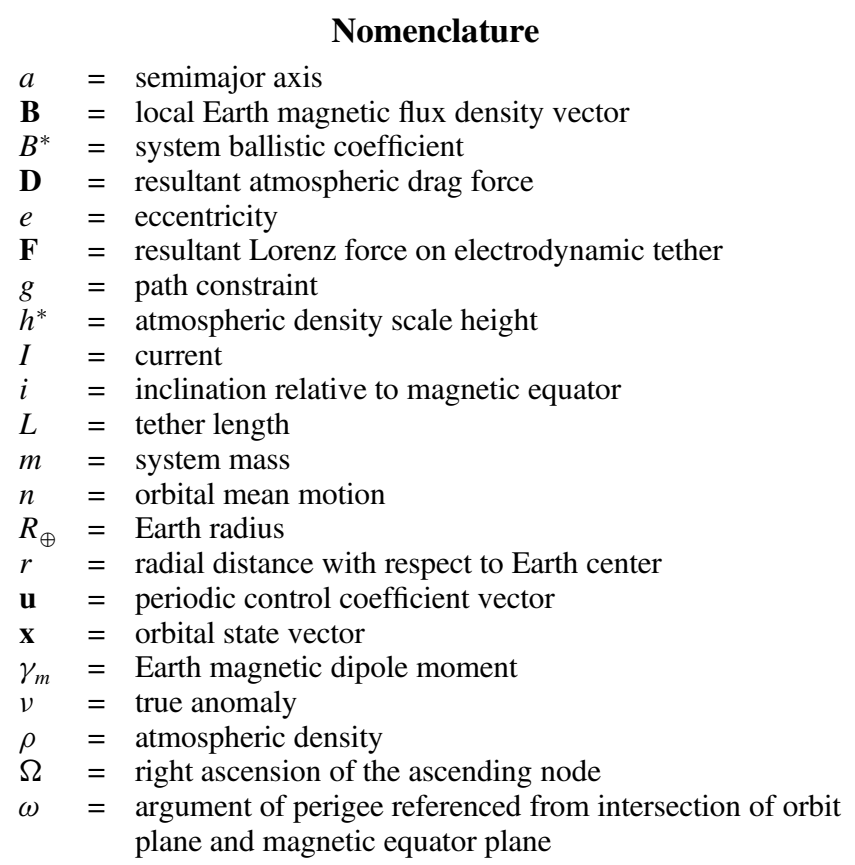

\section{Introduction}

$\mathbf{E}$ LECTRODYNAMIC tethers (EDTs) in low Earth orbit offer an attractive alternative to conventional satellites that use propellant-based propulsion systems because the thrusting forces are derived from the Earth's magnetic field, a renewable resource. Electrodynamic tethers are electrically conductive wires extending between two or more subsatellites. When a current is passed through the wire in the Earth's magnetic field, a Lorenz force is generated

Received 6 November 2007; revision received 1 February 2008; accepted for publication 7 March 2008. This material is declared a work of the U.S. Government and is not subject to copyright protection in the United States. Copies of this paper may be made for personal or internal use, on condition that the copier pay the $\$ 10.00$ per-copy fee to the Copyright Clearance Center, Inc., 222 Rosewood Drive, Danvers, MA 01923; include the code 0731-5090/ $08 \$ 10.00$ in correspondence with the CCC

${ }^{*} \mathrm{Ph} . \mathrm{D}$. Candidate, Department of Aeronautics and Astronautics; robert.stevens@afit.edu. Student Member AIAA.

†Professor, Department of Aeronautics and Astronautics; william.wiesel@ afit.edu. perpendicular to both the current direction and the direction of the local Earth magnetic field lines. A two-ball EDT is depicted in Fig. 1. The force magnitude depends on the current, length of wire, and the wire orientation with respect to the local magnetic field. Controlling the current in the wire through variable resistance, the satellite system would be capable of maneuvering to new orbits without propellant, albeit at a slower rate than traditional maneuvering rockets. Because of the slow rate of change, an orbit transfer requires a long time to reach a desired orbit; thus, a method of control is needed to achieve optimal trajectories that span many orbital revolutions.

Obtaining optimal control solutions for satellites that maneuver over the course of many orbital revolutions can be challenging and computationally intensive when states and controls are considered on short time scales of a few orbital revolutions. Williams demonstrates a method of optimal control using nonlinear perturbation equations of motion as dynamic constraints and solves an optimal control problem by direct transcription using nonlinear programming (NLP) software [1]. This method is shown to be effective in determining controls that execute a modest orbital maneuver using an electrodynamic tether for thrust; however, the solver required hundreds of collocation node points to capture all the small state variations that occur in a single day of maneuvering. This corresponds to thousands of optimization variables and constraints for the NLP solver to compute. The number of nodes and computation time required to perform the optimization over long periods of time can be difficult or impossible to achieve using the short time scale model and are susceptible to round-off errors. In many low-thrust maneuvering situations, the instantaneous orbit state will vary only slightly within an orbital period due to small perturbations, but the variations tend to be periodic in the short term and cancel out over the long term, leaving only slow secular state changes. Carroll [2] and Tragesser and San [3] present a technique of nonoptimal periodic tether control that uses the method of averaging derived from perturbation theory, enabling control of the average states and thus avoiding the computational burden associated with controlling the rapidly changing instantaneous states. They demonstrate that this approach is good for determining controls for longer time periods; however, the results are not optimal, and the periodic control is considered to be unchanging throughout the trajectory. Furthermore, determining the control requires constraining the maximum current, which is less straightforward using the method of averaging than constraining instantaneous current using the short time scale model. The aim of this paper is to take advantage of both control methods to achieve optimal control of an electrodynamic tether over long periods of time. This is 


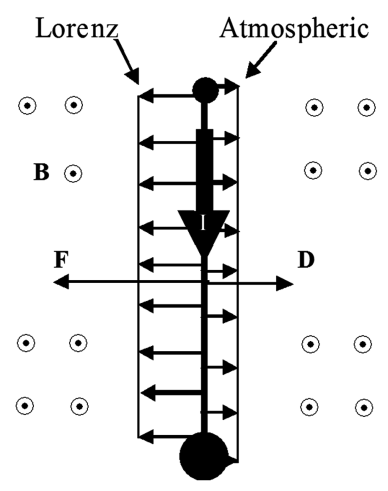

Fig. 1 Electrodynamic tether FBD.

accomplished by recognizing the periodic nature of the optimal control problem and transforming the constraints from the time domain into Fourier space, where the optimization problem is drastically reduced.

The objective is to maneuver a low-thrust satellite system to a new orbit over many revolutions by posing an optimal control problem in the context of large time scales because we are mainly interested in the secular behavior and not the periodic behavior occurring during each revolution. Although any low-thrust system could use this method of large time scale optimal control, propulsion using an electrodynamic tether was chosen for demonstration purposes because it may be continuously operated without propellant for a long time.

In this paper, we examine three different maneuvers that move a two-ball, $4 \mathrm{~km}$ EDT from an initial orbit to a new orbit over many revolutions. Because of the low altitudes considered, all the trajectories account for atmospheric drag. We have taken advantage of the fact that the orbits are nearly circular and have expanded the orbital equations of motion about the very small eccentricity and have ignored second and higher order terms. This assumption is good for orbits with eccentricities less than about 0.01 , keeping errors well within the tolerances of the spectral algorithm. Furthermore, the maneuvers are known to occur over many orbital revolutions, and so the small oscillatory changes in the orbital parameters that are evident over short time scales (within each revolution) are averaged out, leaving only the secular changes that occur over long time durations (many revolutions). The only control we have at our disposal to perform the desired maneuvers is the current in the wire using variable resistance. A close look at the type of control that could contribute significantly to the long-term behavior of the EDT reveals that controls periodic with the orbital rate are the only ones that yield secular changes in the orbital parameters. Other contributions of the controller are averaged out in the long term. Therefore, we assume periodic control current modeled using the relevant terms of a Fourier series:

$$
\begin{aligned}
I= & u_{1}(T)+u_{2}(T) \cos v+u_{3}(T) \sin v \\
& +u_{4}(T) \cos 2 v+u_{5}(T) \sin 2 v
\end{aligned}
$$

where $v$ is the true anomaly. To highlight the fact that the controlled Fourier coefficients vary only over large time scales, we write them as functions of $T$. The control in Fourier space is, therefore,

$$
\mathbf{u}(T)=\left[u_{1}, u_{2}, u_{3}, u_{4}, u_{5}\right]^{T}
$$

With the control written in this form, the approach to optimal control is viewed in the Fourier space, where the goal is to determine the time dependent Fourier coefficients $\mathbf{u}(T)$ that minimize a given cost function for a trajectory subject to the time-averaged dynamic equations of motion. A pseudospectral method of dynamic optimization is employed using DIDO software [4,5] to solve the subject optimal control problems, yielding the optimal control coefficients and path discretized over large periods of time.

\section{Dynamic Model}

Orbital changes due to the relatively weak Lorenz forces generated along the electrodynamic tether occur over many orbital revolutions. The EDT is modeled as a "dumbbell" consisting of two end bodies tethered together with a taut $4 \mathrm{~km}$ copper wire. The Lorenz force generated along the wire containing electric current is given by

$$
\mathbf{F}=I \mathbf{L} \times \mathbf{B}
$$

where $I$ represents tether current (the control), B represents the Earth's local magnetic flux density vector, and $\mathbf{L}$ is the tether length vector pointing in the direction from the upper end mass to the lower one. The tether geometry and current direction that yields a positive transverse thrust is shown in Fig. 1. The local magnetic flux density for an Earth-orbiting satellite is modeled as

$$
\mathbf{B}=\frac{\gamma_{m}}{r^{3}}\left[\begin{array}{c}
-2 \sin (\omega+\nu) \sin i \\
\cos (\omega+\nu) \sin i \\
\cos i
\end{array}\right]_{e}=\left[\begin{array}{c}
B_{r} \\
B_{t} \\
B_{n}
\end{array}\right]_{e}
$$

where $\gamma_{m}$ represents the Earth's magnetic dipole moment, $i$ is the inclination relative to the magnetic equator, and $B_{r}, B_{t}$, and $B_{n}$ represent the magnetic flux density vector components in the radial, transverse, and orbit normal directions, respectively (i.e., $\hat{e}_{r}, \hat{e}_{t}$, and $\hat{e}_{n}$ directions). In the best conditions at the equator, a force of $0.1 \mathrm{~N}$ distributed along a 1-amp, $4 \mathrm{~km}$ EDT is achievable at an altitude of $270 \mathrm{~km}$, which can be the same order of magnitude as the atmospheric drag at that altitude, depending on the physical characteristics of the tether and end bodies. To ensure the satellite lives longer than a few days, the control system will need to apply a constant average current to provide constant in-track thrust that will compensate for drag forces acting in the opposite direction. The problem of drag compensation is exacerbated when the EDT resides in a higher inclination orbit because the out-of-plane component of the magnetic field, which produces the required in-track thrust, is reduced [Eq. (4)]. Drag magnitude depends on the physical properties and dimensions of the EDT, the atmospheric density, and satellite velocity. For a near circular orbit, the drag force on the entire tether system is given by

$$
\mathbf{D}=-\frac{1}{2} B^{*} \rho(r) \frac{\mu}{r} \hat{\mathbf{e}}_{\mathbf{t}}
$$

where $\rho(r)$ represents the average air density at radial distance $r$, and $B^{*}$ is the average ballistic coefficient of the entire tether. Here the ballistic coefficient is defined as $B^{*}=C_{d} A / m$, where $C_{d}$ is the average coefficient of drag, $A$ is the average cross-sectional area perpendicular to the velocity vector, and $m$ is the system mass. Modeling an exponentially decaying atmospheric density using a scale height $h^{*}$, we can expand about the small eccentricity and approximate the average density through first order as []ㅡ

$$
\rho(r)=\rho_{0} e^{-\frac{r-R_{\oplus}}{h^{*}}} \approx \rho(a)\left(1+\frac{a e \cos v}{h^{*}}\right)
$$

where the radial distance has been approximated as $r \approx a(1-e \cos v)$.

Gravity gradient torque tends to keep the tether nadir pointing with librations that are assumed to be small, and so the acceleration due to the Lorenz force in Eq. (3) is given by

$$
\mathbf{F}=\frac{I L \gamma_{m}}{m r^{3}}\left(\cos i \hat{\mathbf{e}}_{\mathbf{t}}-\cos (v+\omega) \sin i \hat{\mathbf{e}}_{\mathbf{n}}\right)
$$

Recognizing that the orbits of interest at this low altitude are nearly circular, we ignore $O\left(e^{2}\right)$ and higher order terms and write the equations of variation for five classical orbital elements as 


$$
\begin{aligned}
\frac{\mathrm{d} a}{\mathrm{~d} t} & =\frac{2 a}{n r}(\mathbf{F}+\mathbf{D}) \cdot \hat{\mathbf{e}}_{\mathbf{t}} \quad \frac{\mathrm{d} e}{\mathrm{~d} t}=\frac{1}{n a^{2} e}\left(\frac{a^{2}}{r}-r\right)(\mathbf{F}+\mathbf{D}) \cdot \hat{\mathbf{e}}_{\mathbf{t}} \\
\frac{\mathrm{d} \omega}{\mathrm{d} t} & =\frac{1}{n a e}\left(1+\frac{1}{1+e \cos v}\right) \sin v(\mathbf{F}+\mathbf{D}) \\
& \cdot \hat{\mathbf{e}}_{\mathbf{t}}-\frac{r \cot i \sin (v+\omega)}{n a^{2}} \mathbf{F} \cdot \hat{\mathbf{e}}_{\mathbf{n}} \\
\frac{\mathrm{d} i}{\mathrm{~d} t} & =\frac{r \cos (v+\omega)}{n a^{2}} \mathbf{F} \cdot \hat{\mathbf{e}}_{\mathbf{n}} \quad \frac{\mathrm{d} \Omega}{\mathrm{d} t}=\frac{r \sin (v+\omega)}{n a^{2} \sin i} \mathbf{F} \cdot \hat{\mathbf{e}}_{\mathbf{n}}
\end{aligned}
$$

where $n$ is the mean motion of the satellite. Expanding these equations of motion about the small eccentricity using $r^{-1} \approx$ $a^{-1}(1+e \cos v)$ and ignoring second and higher order terms, we write the general perturbation equations of motion for a nadirpointing tether in terms of the true anomaly, the only variable that changes significantly on a short time scale.

$$
\begin{aligned}
& \frac{\mathrm{d} a}{\mathrm{~d} t} \approx 2 C a \cos i I(1+4 e \cos v)-2 D\left[1+\left(2+\frac{a}{h^{*}}\right) e \cos v\right] \\
& \frac{\mathrm{d} e}{\mathrm{~d} t} \approx C \cos i I\left(2 \cos v+5 e \cos ^{2} v+e\right)-\frac{2 D}{a}\left[\cos v+e+\left(1+\frac{a}{h^{*}}\right) e \cos ^{2} v\right] \\
& \frac{\mathrm{d} \omega}{\mathrm{d} t} \approx \frac{C \cos i \sin (2 \omega+2 v)}{2} I(1+2 e \cos v) \\
& \quad+\sin v\left\{\frac{C \cos i}{e} I(2+5 e \cos v)-\frac{D}{a e}\left[2+\left(1+\frac{2 a}{h^{*}}\right) e \cos v\right]\right\} \\
& \frac{\mathrm{d} i}{\mathrm{~d} t} \approx-C \sin i I \cos ^{2}(v+\omega)(1+2 e \cos v) \\
& \frac{\mathrm{d} \Omega}{\mathrm{d} t} \approx-\frac{C I}{2} \sin (2 v+2 \omega)(1+2 e \cos v)
\end{aligned}
$$

We have let $C=L \gamma_{m} / n m a^{4}$ represent the term resulting from thrust, and $D=B^{*} \mu \rho(a) / 2 n a$ represent the drag term. In this form, these equations could serve as dynamic constraints in posing our optimal control problems; however, due to the rapid variation of true anomaly with each revolution, we would need to discretize the problem with enough node points for the solver to capture the motion of each varying element with each revolution. This is the approach Williams used in [1] to achieve optimal control solutions for short time scale problems. Because we are only interested in the secular state changes of the EDT orbit over long time scales, we use the method of averaging to eliminate the small oscillations that occur within each revolution, which effectively approximates the nonautonomous system in Eq. (9) as an autonomous averaged one [7]. This is achieved by recognizing that

$$
\mathrm{d} t=\frac{1}{n}\left[1-2 e \cos v+O\left(e^{2}\right)\right] \mathrm{d} v
$$

and then integrating over $2 \pi N$ orbits $(N=1,2, \ldots)$. Because the average states vary slowly with time, they are considered constant over the short time periods of integration and are removed from the integrand. The fast time variable $v$ always appears in the argument of a sine or a cosine function, thus will only yield nonzero values after integration when the current control $I$ is itself a linear combination of sine and cosine functions periodic with $v$ and a constant. A current that is purely dc will produce secular motion in semimajor axis and inclination because the first two derivatives in Eq. (9) would yield nonzero values after integration. Because the orbits of interest remain very close to the Earth, where the magnetic field is strong, they are nearly circular. We will, therefore, substitute two equinoctial coordinates for the eccentricity and argument of perigee in Eq (9) to avoid singularity near $e=0$. The new coordinates are the eccentricity vector components defined as $h=e \sin \omega$ and $k=e \cos \omega$. The average state equations of motion are derived in the appendix using the periodic current defined in Eq. (1), and are written as

$$
\begin{aligned}
& \frac{\Delta a}{\Delta T} \approx 2 C a \cos i\left(u_{1}+u_{2} e\right)-2 D \\
& \frac{\Delta h}{\Delta T} \approx C \cos i\left[\left(\frac{3 h}{2}\right) u_{1}+\left(\frac{h}{e}\right) u_{2}+\left(\frac{k}{e}\right) u_{3}+\left(\frac{h}{4}+\frac{h k^{2}}{2 e^{2}}\right) u_{4}\right. \\
& \left.\quad+\left(\frac{k}{4}+\frac{\left(k^{2}-h^{2}\right) k}{4 e^{2}}\right) u_{5}\right]-\frac{D}{a}\left(1+\frac{a}{h^{*}}\right) h \\
& \frac{\Delta k}{\Delta T} \approx C \cos i\left[\left(\frac{3 k}{2}\right) u_{1}+\left(\frac{k}{e}\right) u_{2}-\left(\frac{h}{e}\right) u_{3}+\left(\frac{k}{4}-\frac{h^{2} k}{2 e^{2}}\right) u_{4}\right. \\
& \left.\quad+\left(-\frac{h}{4}+\frac{\left(h^{2}-k^{2}\right) h}{4 e^{2}}\right) u_{5}\right]-\frac{D}{a}\left(1+\frac{a}{h^{*}}\right) k \\
& \frac{\Delta i}{\Delta T} \approx-C \sin i\left[\left(\frac{1}{2}\right) u_{1}+\left(\frac{k^{2}-h^{2}}{4 e^{2}}\right) u_{4}-\left(\frac{h k}{2 e^{2}}\right) u_{5}\right] \\
& \frac{\Delta \Omega}{\Delta T} \approx-C\left[\left(\frac{h k}{2 e^{2}}\right) u_{4}+\left(\frac{k^{2}-h^{2}}{4 e^{2}}\right) u_{5}\right]
\end{aligned}
$$

Secular changes to the orbit state are now expressed over a large time scale $\Delta T=2 \pi N / n$. The state vector $\mathbf{x}$ now represents the average orbital state values rather than the instantaneous values and is written using a quasi-equinoctial element set, that is, $\mathbf{x}(T)=[a, h, k, i, \Omega]^{T}$. Notice that these average states only vary slowly over long time scales (indicated by $T$ ) and are considered constant within each revolution. The average state equations of motion are thus devoid of the short time scale variable, the true anomaly. From the first equation, we see that the average drag effect due to the air density (in the drag term $D$ ) primarily affects the average change in semimajor axis. To a lesser extent, drag decreases the $h$ and $k$ states and has a circularizing effect because $e=\sqrt{h^{2}+k^{2}}$. With the secular equations of motion in hand, we now turn to constraining the allowable tether current to values that are within the system power limitations.

\section{Constraints}

To determine the optimal controls for the system described by Eq. (11), we need to solve for the periodic control coefficients $\mathbf{u}(T)$. In addition to enforcing the initial state conditions as event constraints, the control current must also be bound to remain within an available power limit, which is itself defined by the electron collection capabilities, ohmic losses, voltage current, and other factors. For a description of electron collection in the ionosphere and the associated limitations, see [8]. Because the control in Eq. (1) is defined using the rapidly changing true anomaly, we cannot simply bound the instantaneous periodic current between a minimum and maximum value because we need to keep our averaged equations of motion devoid of short time scale variables. To properly bound the control then, we need to define a path constraint that is a function of the slowly varying Fourier control coefficients $\mathbf{u}(T)$. The approach used here limits the average power available for thrust which in turn places bounds the on the rms current. For a given constant wire resistance $R$ and average power limit $P_{\text {avgmax }}$, the maximum allowable rms current is defined by

$$
I_{\mathrm{rms} \max }^{2}=\frac{P_{\mathrm{avg} \max }}{R}
$$

The actual rms value for the periodic current in the wire is defined by

$$
I_{\mathrm{rms}}^{2}=\frac{1}{2 \pi} \int_{0}^{2 \pi} I^{2}(\mathbf{u}, \nu) \mathrm{d} \nu
$$

For the periodic current given in Eq. (1), this value is

$$
I_{\mathrm{rms}}^{2}=u_{1}^{2}+\frac{1}{2}\left(u_{2}^{2}+u_{3}^{2}+u_{4}^{2}+u_{5}^{2}\right)
$$

Using Eq. (14), we may express the path constraints in terms of the controls. The path constraint for the controls is written as

$$
g_{1}[\mathbf{u}(T)]=I_{\mathrm{rms}}^{2}-\frac{P_{\mathrm{avg} \max }}{R} \leq 0
$$


This path constraint approach has the double advantage of averaging out any parameters periodic with the orbit that affect the available thrust current, such as diurnally varying ionospheric electron density, as well as eliminating the short time variable, the true anomaly. The event constraints (constraints on states at specific times during the trajectory) are comprised only of the initial conditions and are written as

$$
\mathbf{e}\left[\mathbf{x}\left(T_{0}\right)\right]=\left[a\left(T_{0}\right), h\left(T_{0}\right), k\left(T_{0}\right), i\left(T_{0}\right)\right]^{T}
$$

Finally, states, controls, and time are bounded by upper and lower limits (denoted using subscripts $u$ and $l$, respectively). These box constraints are written as

$$
\mathbf{x}_{l} \leq \mathbf{x}(T) \leq \mathbf{x}_{u} \quad \mathbf{u}_{l} \leq \mathbf{u}(T) \leq \mathbf{u}_{u} \quad T_{0 l} \leq T_{0} \leq T_{0 u} \quad T_{f l} \leq T_{f} \leq T_{f u}
$$

Now all the pieces are in place to construct and solve optimal control problems that will maneuver an EDT to a new orbit over many revolutions while overcoming drag by controlling nothing but current in a wire.

\section{Three Optimal Control Problems and Their Solutions}

Three sample maneuvers were chosen to demonstrate large time scale optimal control because of their slow secular orbital changes that occur over many revolutions. The tether modeled in all three problems is $4 \mathrm{~km}$ long and $2 \mathrm{~mm}$ in diameter. The system mass and average cross-sectional area is $500 \mathrm{~kg}$ and $8 \mathrm{~m}^{2}$, respectively. The first two subsections outline the optimal control problem setup, solution, and results for maximizing inclination and altitude and serve as benchmark problems because other authors have investigated these problems [2,3]. The third subsection provides an example optimal control problem and solution that achieves a minimum time orbit change in average semimajor axis and inclination occurring over 500 revolutions using only 40 nodes in the discretized optimization problem. All problems were solved using
DIDO, an optimization software package that discretizes and solves general optimization problems using a pseudospectral method [9]. Verification of the optimal control solution was achieved by evaluating the Hamiltonian output by DIDO. To demonstrate the accuracy of the model used as the dynamic constraint in these problems, the output Fourier coefficient controls were converted into the time domain and then used to propagate instantaneous states using Eq. (ㅁ).

\section{A. Maximum Final Altitude}

Perhaps there is a need to tow an object (spacecraft, debris, etc.) to a higher orbit in the same orbital plane using an EDT. For the sake of testing the algorithm against a known solution, we seek the maximum altitude an EDT can reach in 50 orbital revolutions with no drag. In this case, we expect that a direct current in the nadir-pointing tether will provide maximum thrust in the direction of the velocity to spiral the spacecraft out to a higher orbit $[2,3]$. Although we may actually want to control the other orbital elements to a desired end state, we seek only this known solution for this benchmark problem. The optimal control problem is written as the following.

Minimize cost

$$
J=-a_{f}
$$

Subject to

$$
\begin{array}{lrl}
\text { dynamic constraints } & \dot{\mathbf{x}}(T)=\mathbf{f}[\mathbf{x}(T), \mathbf{u}(T)] \\
\text { event constraints } & \mathbf{e}\left[\mathbf{x}\left(T_{0}\right)\right]=\left[6648 \mathrm{~km}, 0,0.001,30^{\circ}\right]^{T} \\
\text { path constraints } & g_{1}[\mathbf{u}(T)]=I_{\mathrm{rms}}^{2}-2.25 \leq 0 \mathrm{amps}^{2}
\end{array}
$$

where $\dot{\mathbf{x}}(T)$ is the average state change and $\mathbf{f}[\mathbf{x}(T), \mathbf{u}(T)]=$ $\Delta \mathbf{x} / \Delta T$. Box constraints in Eq. (17) are also enforced where we have chosen the bounds to be

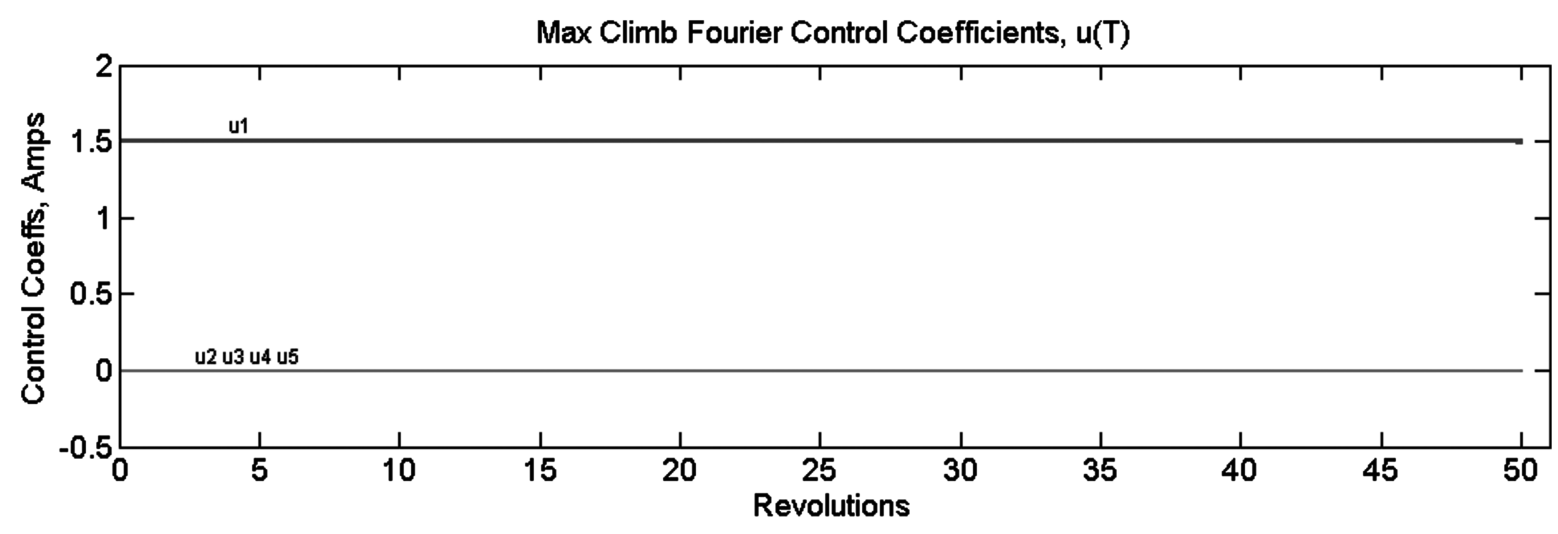

Control in Time Domain

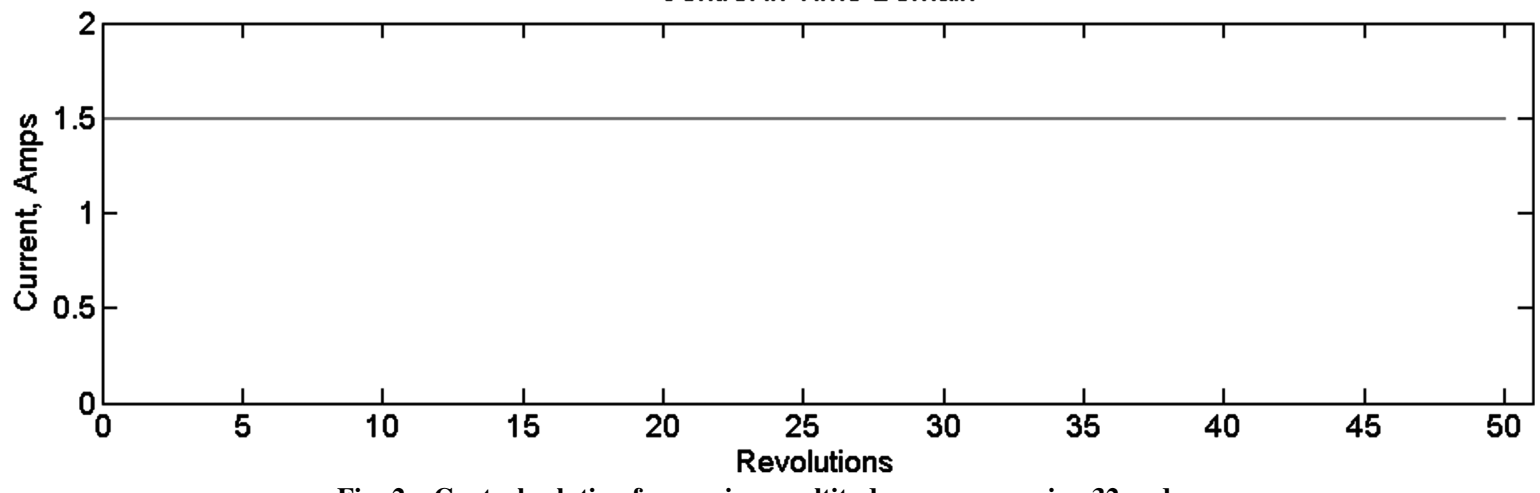

Fig. 2 Control solution for maximum altitude maneuver using 32 nodes. 

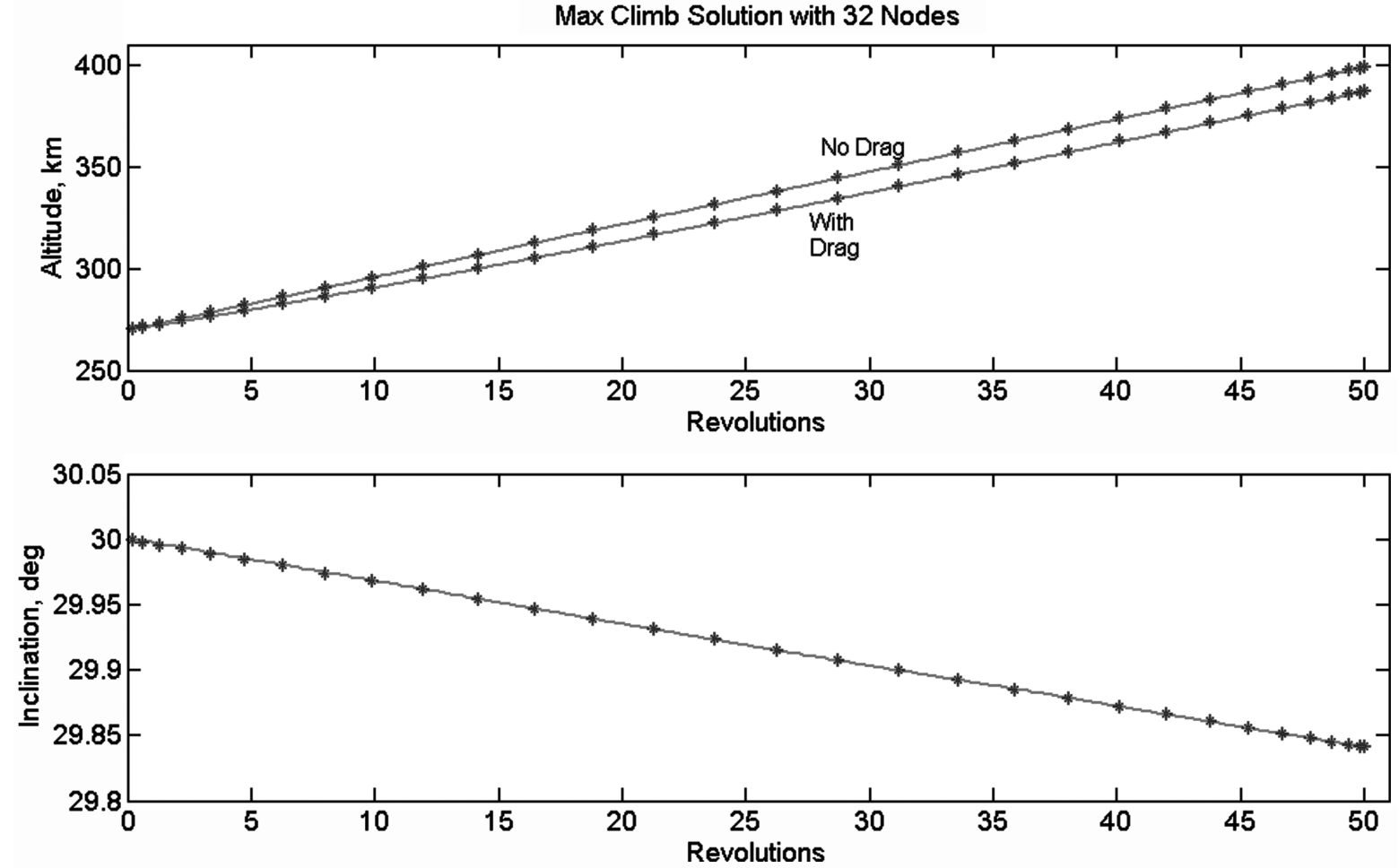

Fig. 3 Maximum altitude maneuver trajectories. Stars indicate DIDO solution; lines indicate instantaneous state propagation using optimal control.

$$
\begin{aligned}
& \mathbf{x}_{u}=[16,000 \mathrm{~km}, 0.4,0.4,80 \mathrm{deg}, 180 \mathrm{deg}] \\
& \mathbf{x}_{l}=[6638 \mathrm{~km},-0.4,-0.4,15 \mathrm{deg},-180 \mathrm{deg}] \\
& \mathbf{u}_{u}=I_{\text {rms max }}[1, \sqrt{2}, \sqrt{2}, \sqrt{2}, \sqrt{2}] \quad \mathbf{u}_{l}=-\mathbf{u}_{u} \quad T_{0}=0 \\
& T_{f}=50 \mathrm{P}
\end{aligned}
$$

where $P$ is the orbital period. The initial states $h$ and $k$ correspond to an eccentricity of 0.001 and an initial argument of perigee of zero. Before using the optimization solver, the states and time were scaled to span values of order 1 to make the problem numerically well conditioned $[4,10]$. Solving the problem using DIDO yields the control history shown at the top of Fig. 2 , and the bottom of the figure shows the control transformed into the short time scale domain, in this case just a direct current. The average altitude and inclination trajectories are shown in Fig. 3 , in which the stars indicate the DIDO solution at discrete times (spanning large time scale steps), and the lines indicate the propagation of the instantaneous state values using DIDO-derived controls and a MATLAB ${ }^{\circledR}$ stiff ordinary differential equation solver. As expected, to perform a maximum climb maneuver, the solution indicates that the controller should drive a maximum allowable direct current through the wire to accomplish the large transverse thrust needed to boost the orbit. Starting at an altitude of $270 \mathrm{~km}$, this EDT can climb about $130 \mathrm{~km}$ in about 3 days without drag. Introducing drag into the dynamic constraints does not affect the control profile but reduces the achievable altitude change in the given time to about $117 \mathrm{~km}$. In reality, we would need to contend with libration control and, at times, adverse electron collection or battery conditions that could limit power available for tether thrusting. However, in principle, modest maneuvers can be accomplished if they are not time critical.

Because there is no explicit time dependence in the Lagrangian of the Hamiltonian of this optimal control problem [Eq. (19)], the resulting Hamiltonian should be constant, that is, $\dot{H}=0$. The Lagrangian of the Hamiltonian is

$$
\bar{H}=H+\mu_{g} g_{1}+\boldsymbol{\mu}_{x}^{T} \mathbf{x}+\boldsymbol{\mu}_{u}^{T} \mathbf{u}
$$

where the Hamiltonian is given by $H=\lambda^{T} \mathbf{f}$ and $\lambda$ represents the costates. The covector functions associated with the path constraint, state-variable box constraints, and control-variable constraints are represented by $\mu_{g}, \boldsymbol{\mu}_{x}$, and $\boldsymbol{\mu}_{u}$, respectively. DIDO uses the covector mapping principle [11] to produce adjoints and the Hamiltonian as part of the solution. To check optimality, we plotted the output Hamiltonian and discovered that it was indeed constant, as shown in Fig. $\underline{4}$.

\section{B. Maximum Final Inclination}

From Eq. (11), it is evident that a carefully and constantly applied dc control current could indeed compensate precisely for drag to maintain altitude; however, it would come at the expense of a secular decay of the inclination after a long time, which may be undesirable. To maximize the final inclination achievable in a fixed time (500 revolutions), we write the same optimal control problem as in the previous example with the following exceptions.

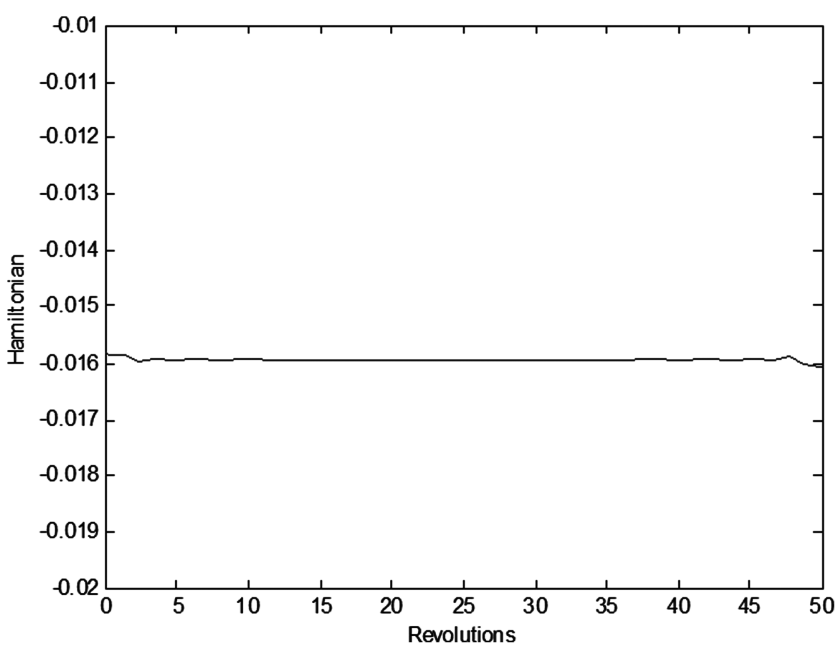

Fig. 4 Hamiltonian profile for maximum altitude with drag solution. 


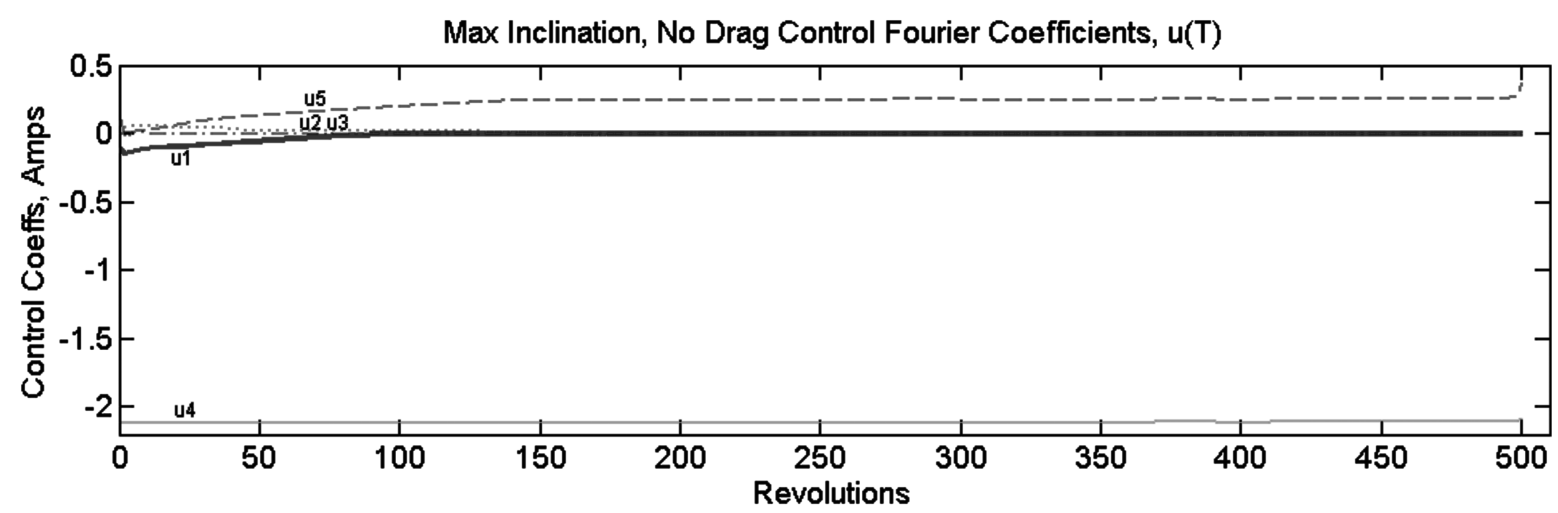

Control in Time Domain

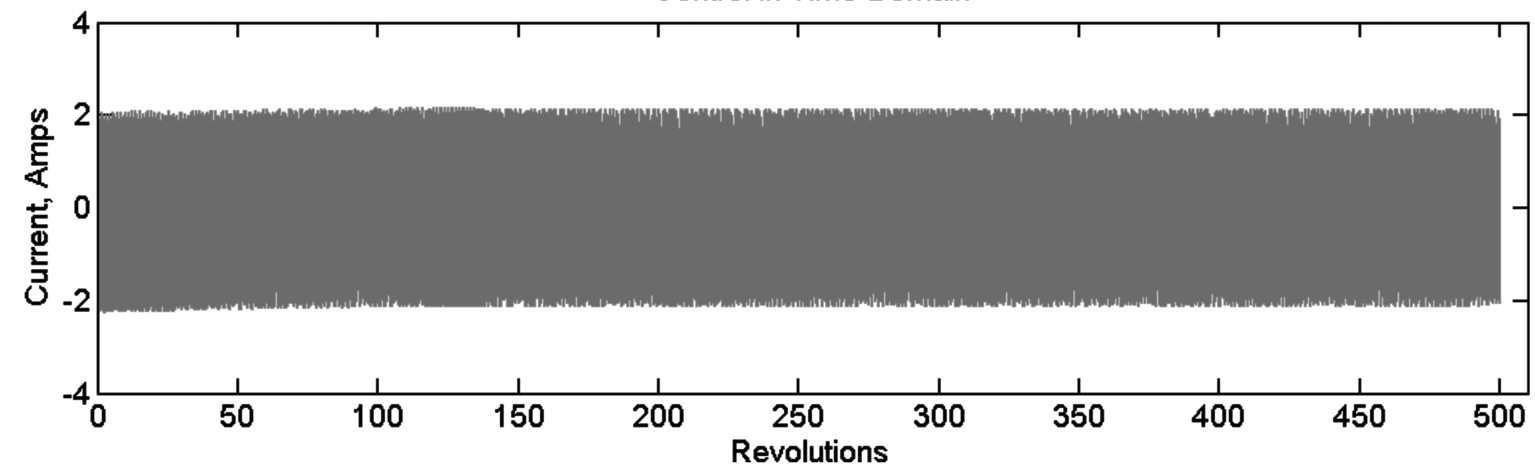

Fig. 5 Maximum inclination control solution with no drag.
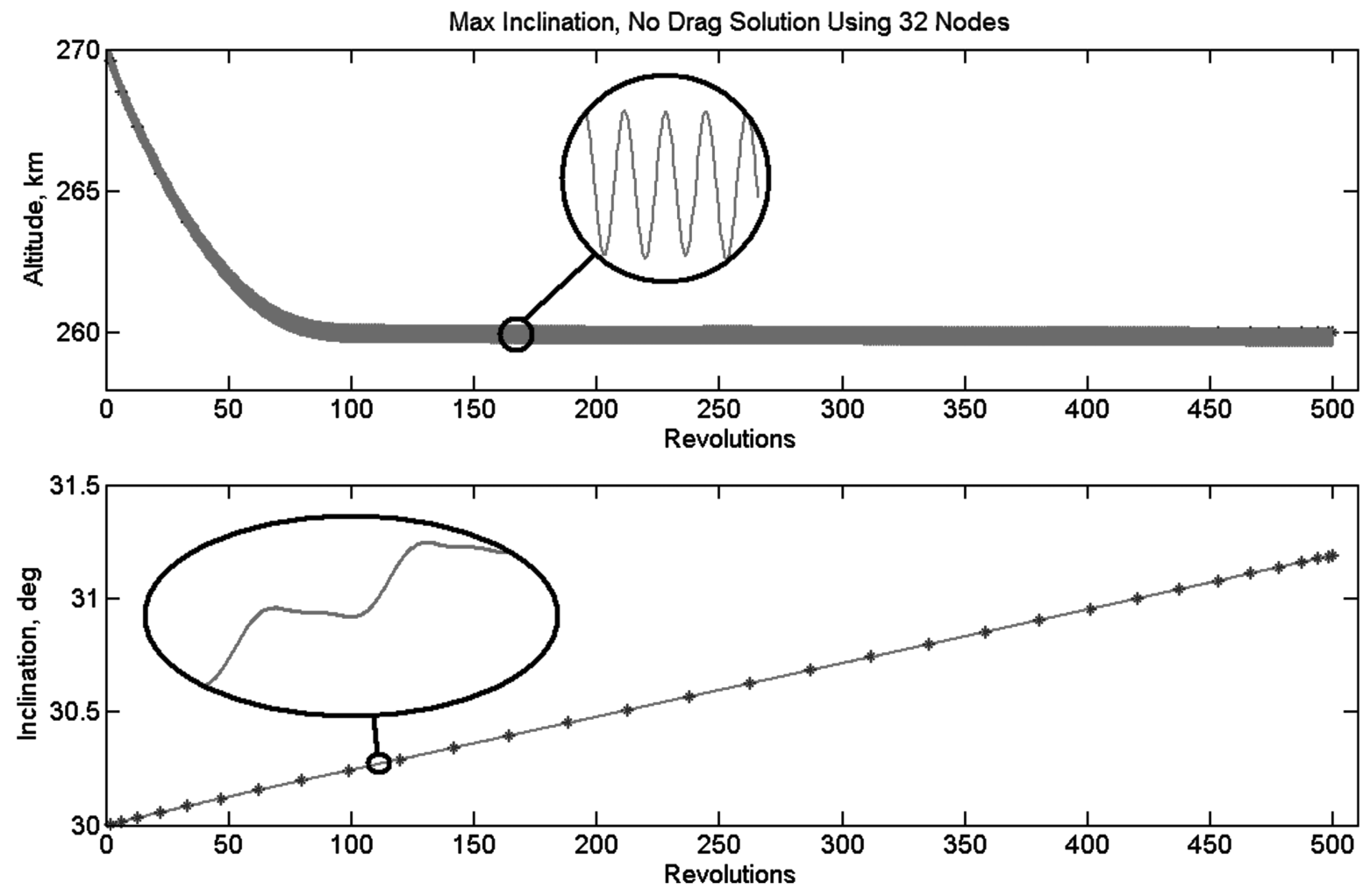

Fig. 6 Maximum inclination maneuver trajectory without drag.

Minimize $\quad J=-i_{f}$

Subject to

$$
\begin{aligned}
& T_{f}=500 P, \\
& \mathbf{e}\left[\mathbf{x}\left(T_{0}\right)\right]=[6648 \mathrm{~km}, 0,0.01,30 \mathrm{deg}]^{T}, \\
& g_{2}[\mathbf{x}(T)]=h^{2}+k^{2}-e_{0}^{2}=0
\end{aligned}
$$

where the new path constraint, $g_{2}[\mathbf{x}(T)]$, ensures a constant eccentricity transfer.

As a test case, we first look for the no drag solution [i.e., atmospheric density terms in Eq. (11) are zero] and then compare it with the solution that accounts for drag. The 32-node DIDO control solution to the no-drag problem is depicted in Fig. 5. The contrast between the two plots of the same control in Fig. 5 clearly shows the advantage of solving the optimal control problem using Fourier 


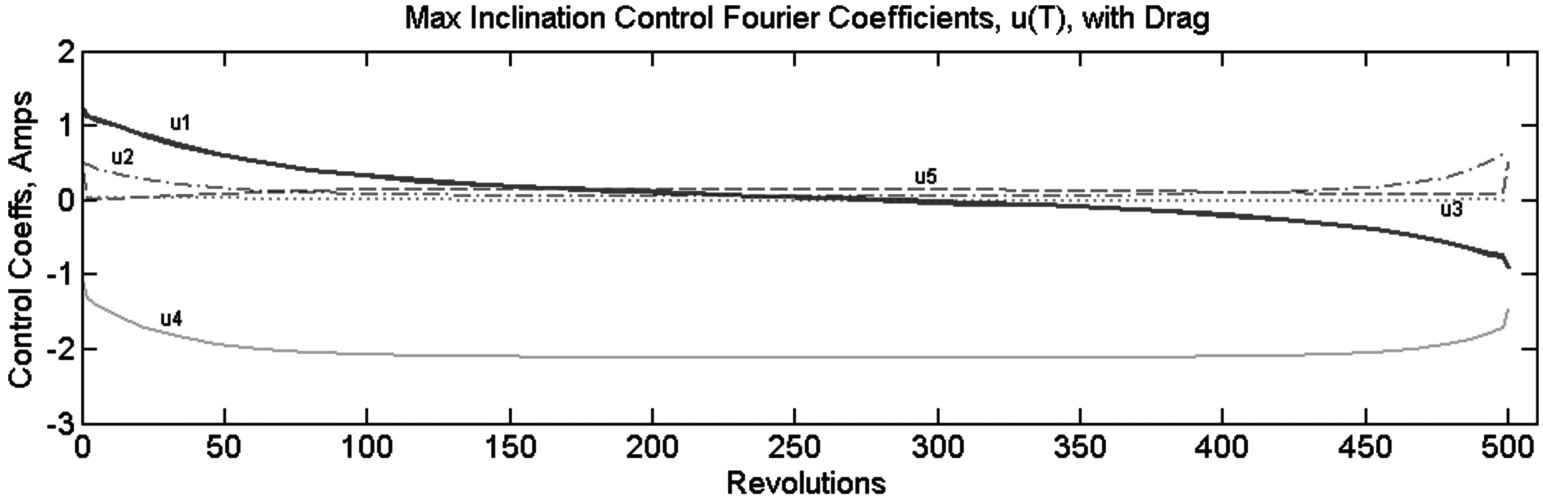

Control in Time Domain

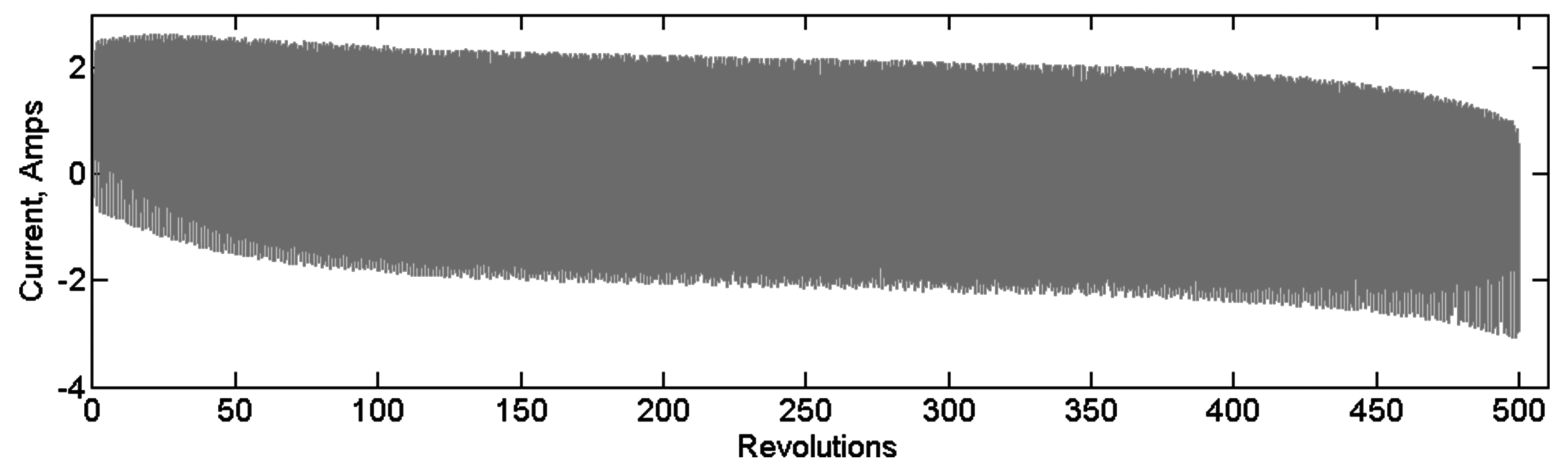

Fig. 7 Maximum inclination control solution with drag.

coefficients over a large time scale. Attempts to discretize and optimize this control problem using instantaneous states and their respective dynamic equations of motion [Eq. (8)] for this long-term trajectory would require thousands of nodes and run the risk of round-off errors and long solution times. Propagating the instantaneous states using the optimal control output produces the trajectory shown in Fig. $\underline{6}$. Because there is no drag to contend with, the optimal solution indicates that it is best to mainly use an ac current that has double the orbital frequency, that is, a combination of $u_{4}$ and $u_{5}$ within constraints. This result is consistent with references $[2,3]$, which indicate that to achieve a maximum inclination change, the strategy is to drive a control current such that $I=-\sqrt{2} I_{\text {rms max }} \cos 2(v+\omega)$. Here, it is assumed that the path constraint in Eq. (15) is active, which bounds the peak amplitude of
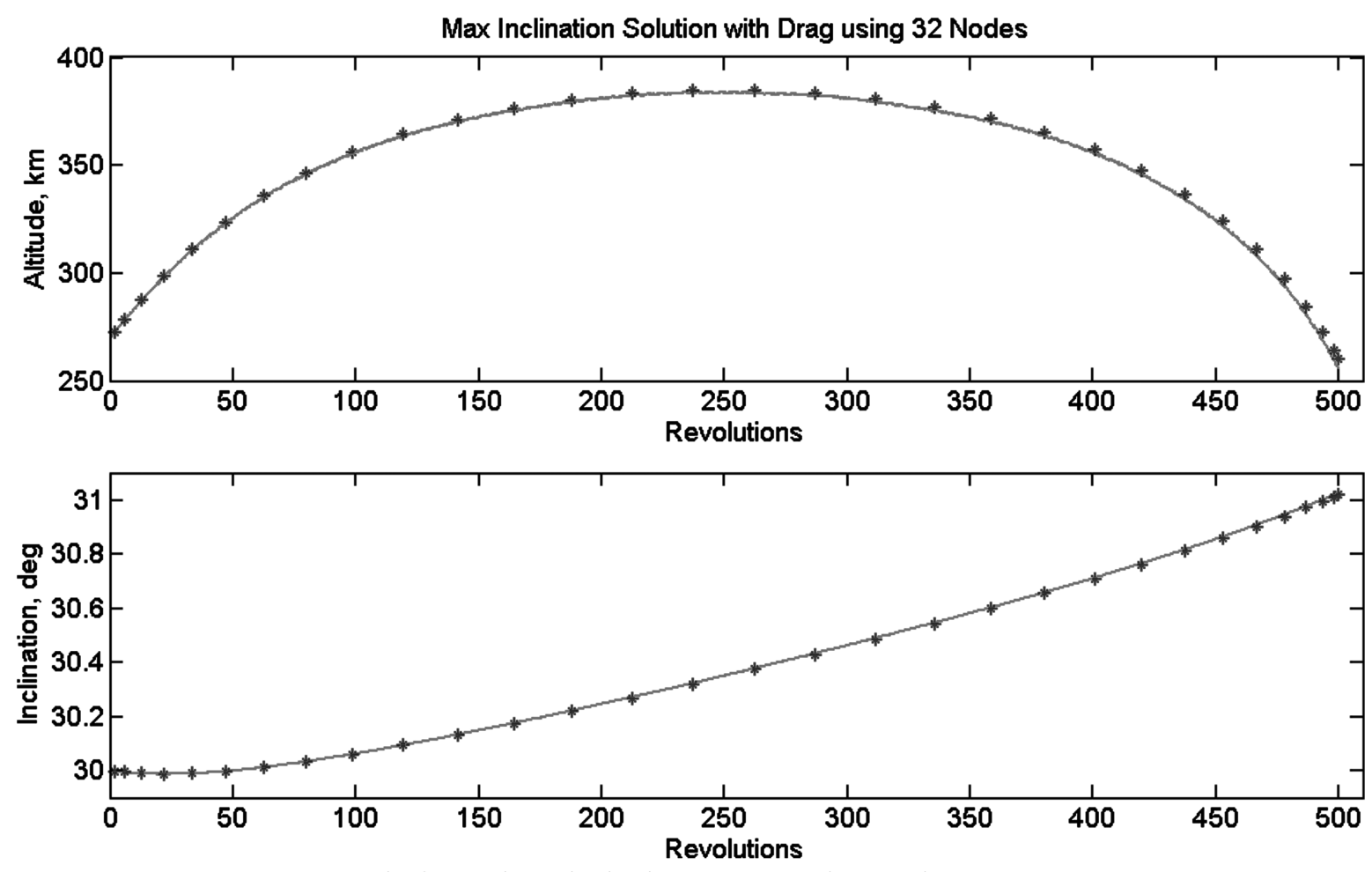

Fig. 8 Maximum inclination maneuver trajectory with drag. 


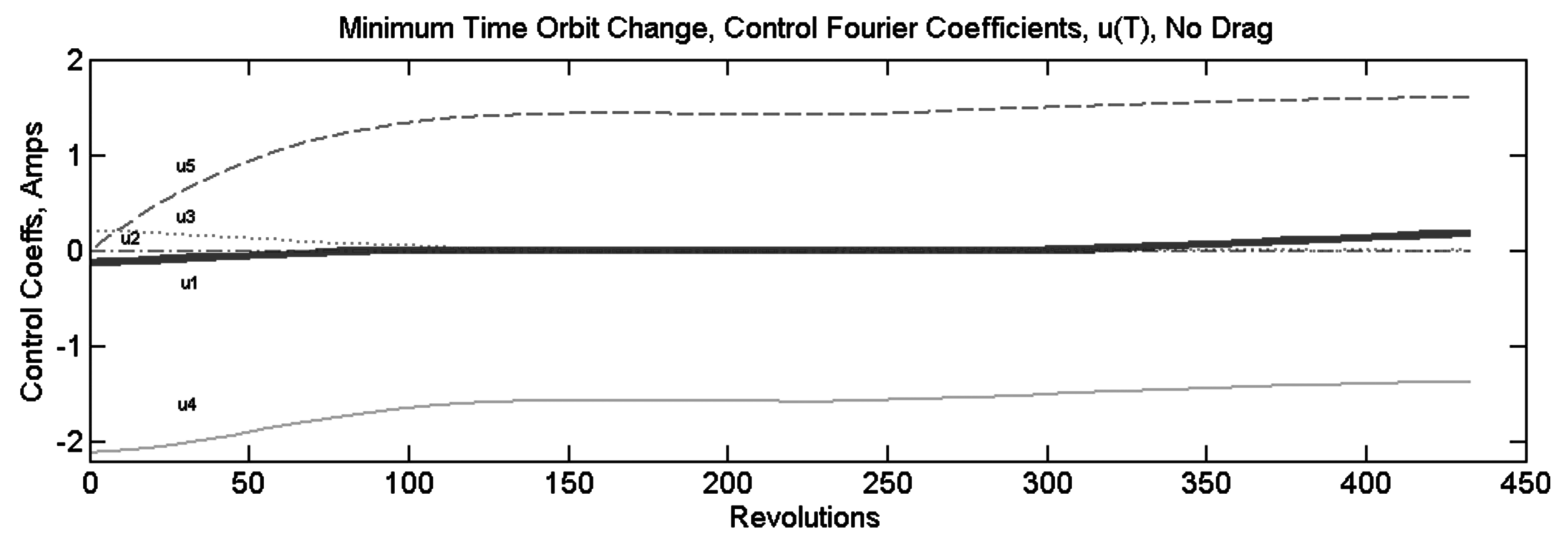

Control in Time Domain

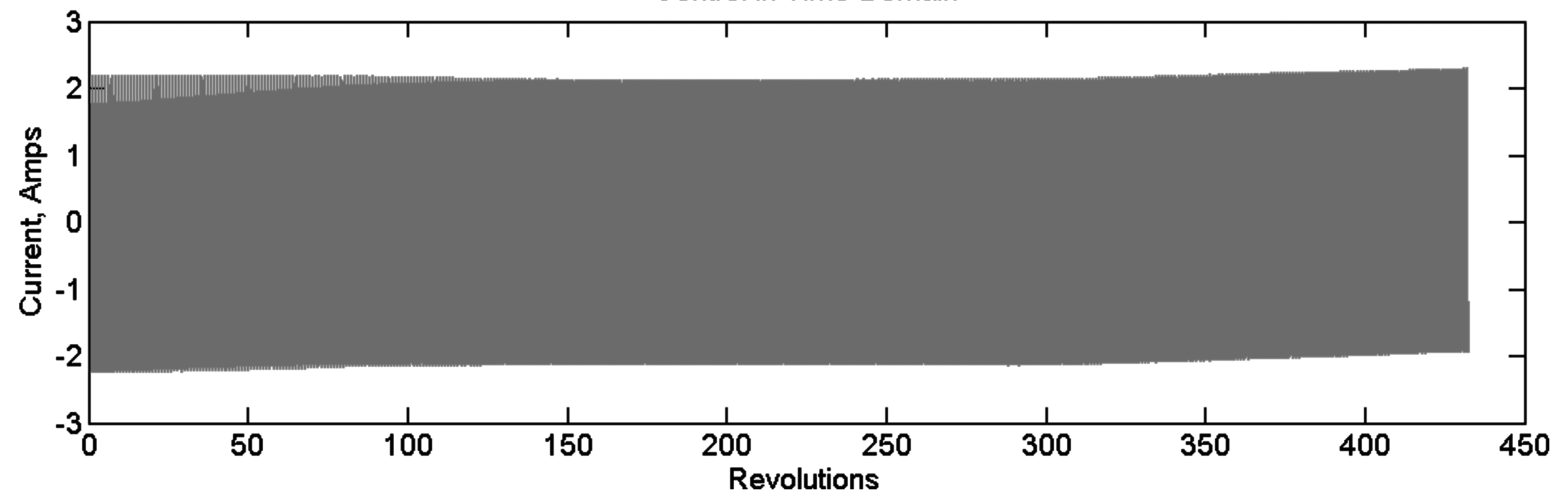

Fig. 9 Minimum time orbit change control solution without drag.

this ac input to $\sqrt{2} I_{\text {rms max }}$. Transforming this result into the Fourier coefficient controller described in Eq. (1), we see that the control solution is the same, only expressed in the context of the partial equinoctial set. To achieve a maximum final inclination, the control may be written

$$
\begin{aligned}
I= & -\sqrt{2} I_{\text {rms max }} \cos (2 v+2 \omega) \\
& =-\sqrt{2} I_{\text {rms max }}(\cos 2 \omega \cos 2 v-\sin 2 \omega \sin 2 v) \\
& =-\sqrt{2} I_{\text {rms max }}\left(\frac{k^{2}-h^{2}}{e^{2}} \cos 2 v-\frac{2 k h}{e^{2}} \sin 2 v\right)
\end{aligned}
$$
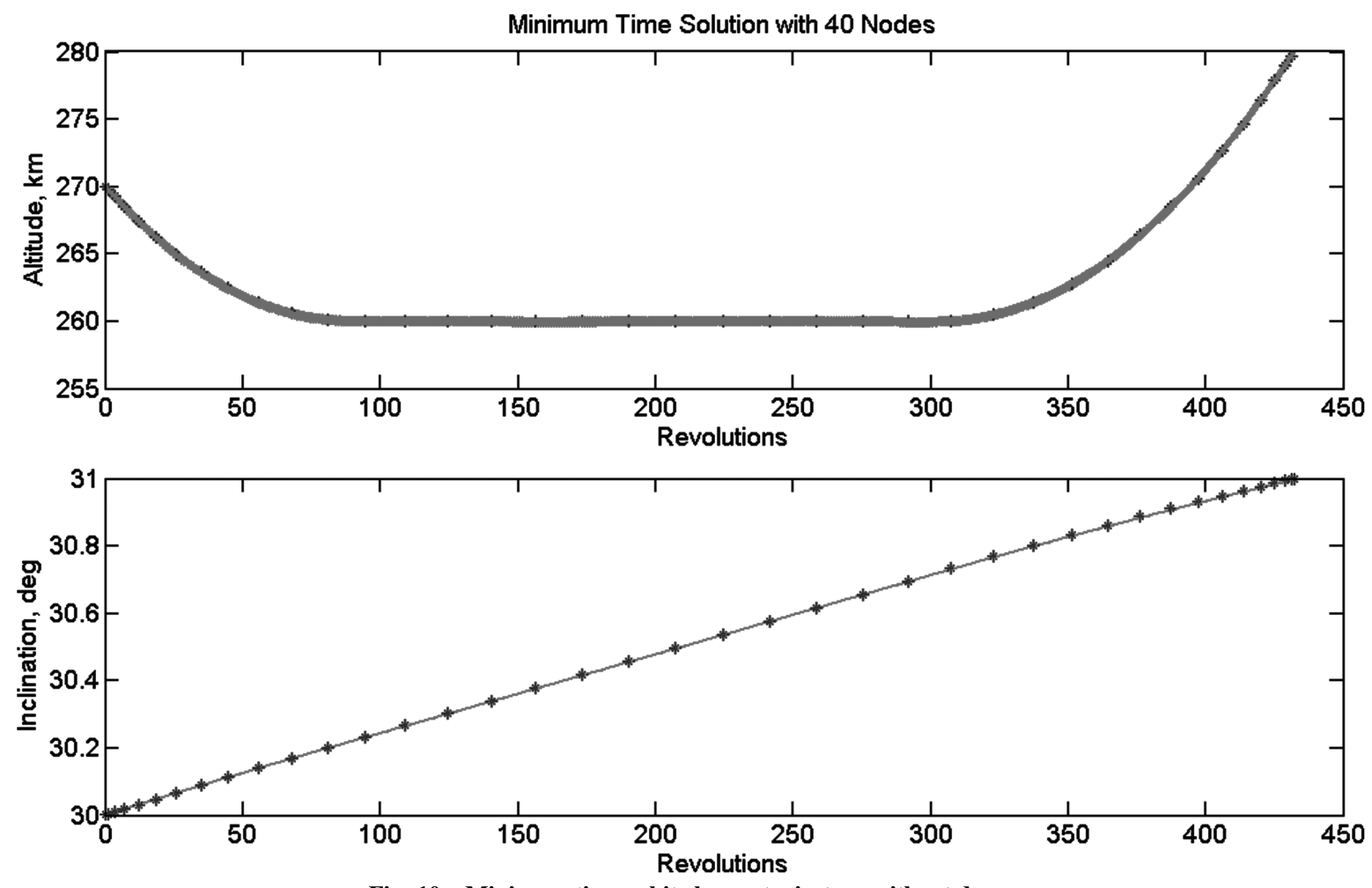

Fig. 10 Minimum time orbit change trajectory without drag. 
In this form, we recognize the Fourier coefficients for the second mode cosine and sine functions as

$$
u_{4}=-\sqrt{2} I_{\mathrm{rms} \max }\left(\frac{k^{2}-h^{2}}{e^{2}}\right) \quad u_{5}=\sqrt{2} I_{\mathrm{rms} \max }\left(\frac{2 k h}{e^{2}}\right)
$$

The optimal controls calculated using Eq. (21) are consistent with the corresponding control Fourier coefficients determined by DIDO (within an error 2 norm of 0.04 ). This trajectory uses some negative $\mathrm{dc}$ thrust to decrease altitude while cranking the orbit to a higher inclination. The Fourier control coefficients displayed in Fig. 5 show that the tether current controller initially uses a small negative dc component to descend to the lowest allowable altitude to maximize the final inclination. Controlling the spacecraft in such a way increases the orbit inclination from 30 to $31.19 \mathrm{deg}$ in about a month. This strategy outperforms a similar constant altitude maneuver by $0.04 \mathrm{deg}$. When drag is considered, the control strategy is altogether different because more of the limited available current must be constant dc to compensate for the increased drag as seen in Fig. 7. We see from Eq. (11) that a large positive dc coefficient tends to reduce the inclination. There is a penalty for orbiting where the air density is higher because more power is expended simply to maintain altitude, which causes inclination to decay and less power to be available to maximize the inclination. In this case, the strategy is to climb to a lower density altitude, level off to increase inclination, then descend again to the minimum allowable altitude, taking advantage of the largest possible inclination gain opportunities as shown in Fig. 8. The initial climb comes at the expense of inclination gain; however, overall the satellite achieves maximum inclination change because it operates in a lower average drag environment and does not need to expend as much power to maintain altitude. After a month of thrusting in a reduced average drag environment, the satellite achieves an inclination gain of $1.0 \mathrm{deg}$ outperforming a constant altitude maneuver by $0.25 \mathrm{deg}$. Because this maneuver occurs over so many revolutions, it would be nearly impossible for short time scale optimization to yield a solution to this problem.

\section{Minimum Time Orbit Change}

Having looked at the baseline orbital maneuvers, we now turn our attention to determining the controls for a minimum time orbit change involving a desired final altitude and inclination while maintaining a constant eccentricity. In this example, we start by using our initial states from the first example and then construct the optimal control problem to achieve a $10 \mathrm{~km}$ climb and a $1 \mathrm{deg}$ inclination increase, while maintaining a constant eccentricity of 0.005 , in the quickest time. We write the problem as follows.

Minimize cost:

$$
J=t_{f}
$$

Subject to

$$
\begin{aligned}
& \dot{\mathbf{x}}(T)=\mathbf{f}[\mathbf{x}(T), \mathbf{u}(T)] \\
& \mathbf{e}_{\mathbf{0}}\left[\mathbf{x}\left(T_{0}\right)\right]=[6648 \mathrm{~km}, 0,0.005,30 \mathrm{deg}]^{T} \\
& \mathbf{e}_{\mathbf{f}}\left[\mathbf{x}\left(T_{f}\right)\right]=\left[a_{f}, i_{f}\right]^{T}=[6658 \mathrm{~km}, 31 \mathrm{deg}]^{T} \\
& g_{1}[\mathbf{u}(T)]=I_{\mathrm{rms}}^{2}-2.25 \leq 0 \mathrm{amps}^{2} \\
& g_{2}[\mathbf{x}(T)]=h^{2}+k^{2}-e_{0}^{2}=0
\end{aligned}
$$

Box constraints are still those listed in relations (17) and Eqs. (18), but because this problem has a free final time, we write

$$
T_{0}+\varepsilon \leq T_{f} \leq 5 \times 10^{4} \mathrm{P}
$$

The control solution without drag, depicted in Fig. 9, indicates that the strategy is to initially apply a negative dc control current, indicated by $u_{1}$, to descend. The controller needs to apply large ac control components cycling at twice the orbital frequency to reach the desired inclination (i.e., large $u_{4}$ and $u_{5}$ components), all while avoiding large components cycling at the orbital frequency, namely $u_{2}$ and $u_{3}$, which are large contributors to eccentricity change. The dc component is nearly zero for the majority of the trajectory and then reverts to positive flow at the end of the trajectory to climb to the final desired orbital altitude (Fig. 10). When drag is considered, the dc
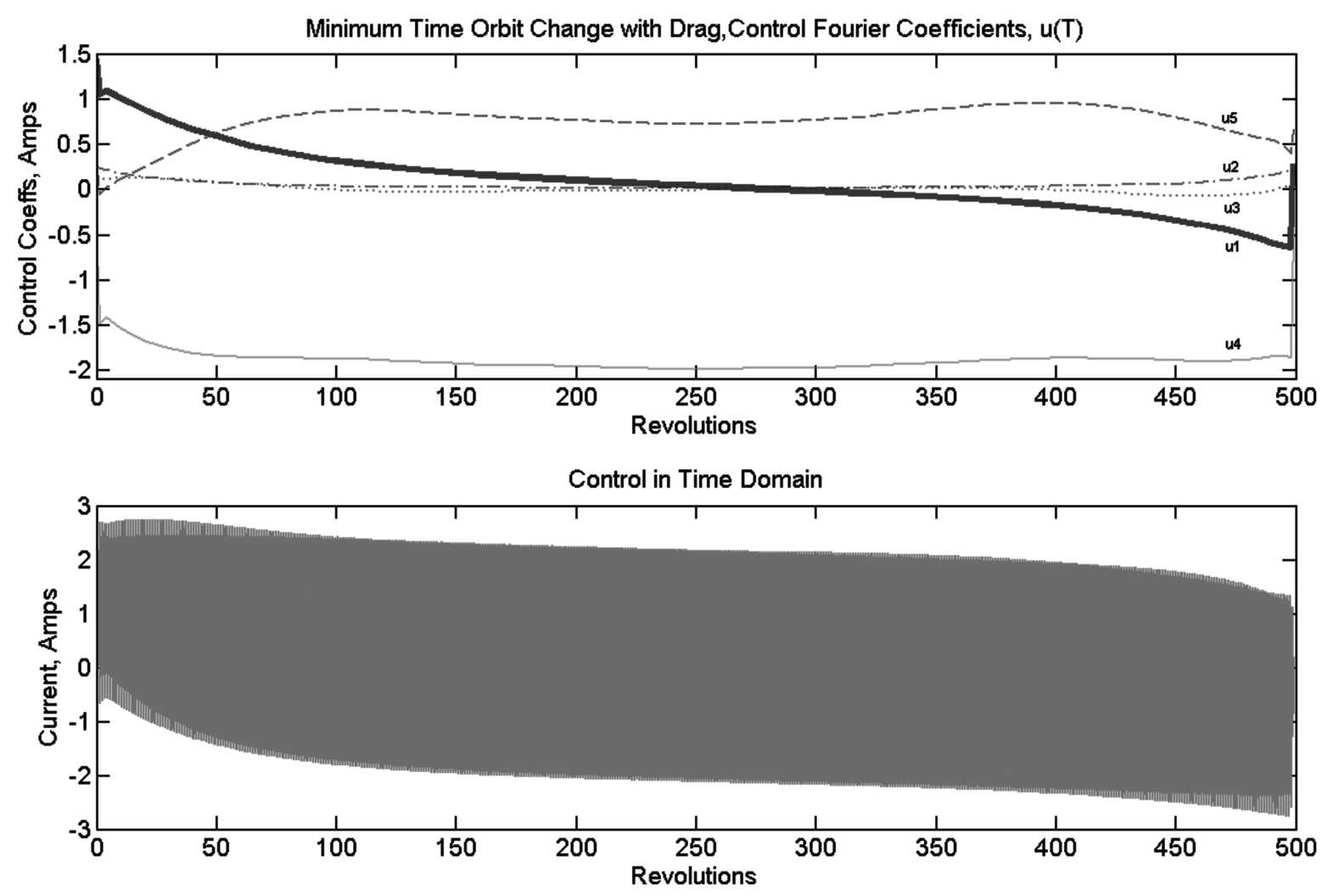

Fig. 11 Minimum time orbit change control solution with drag. 

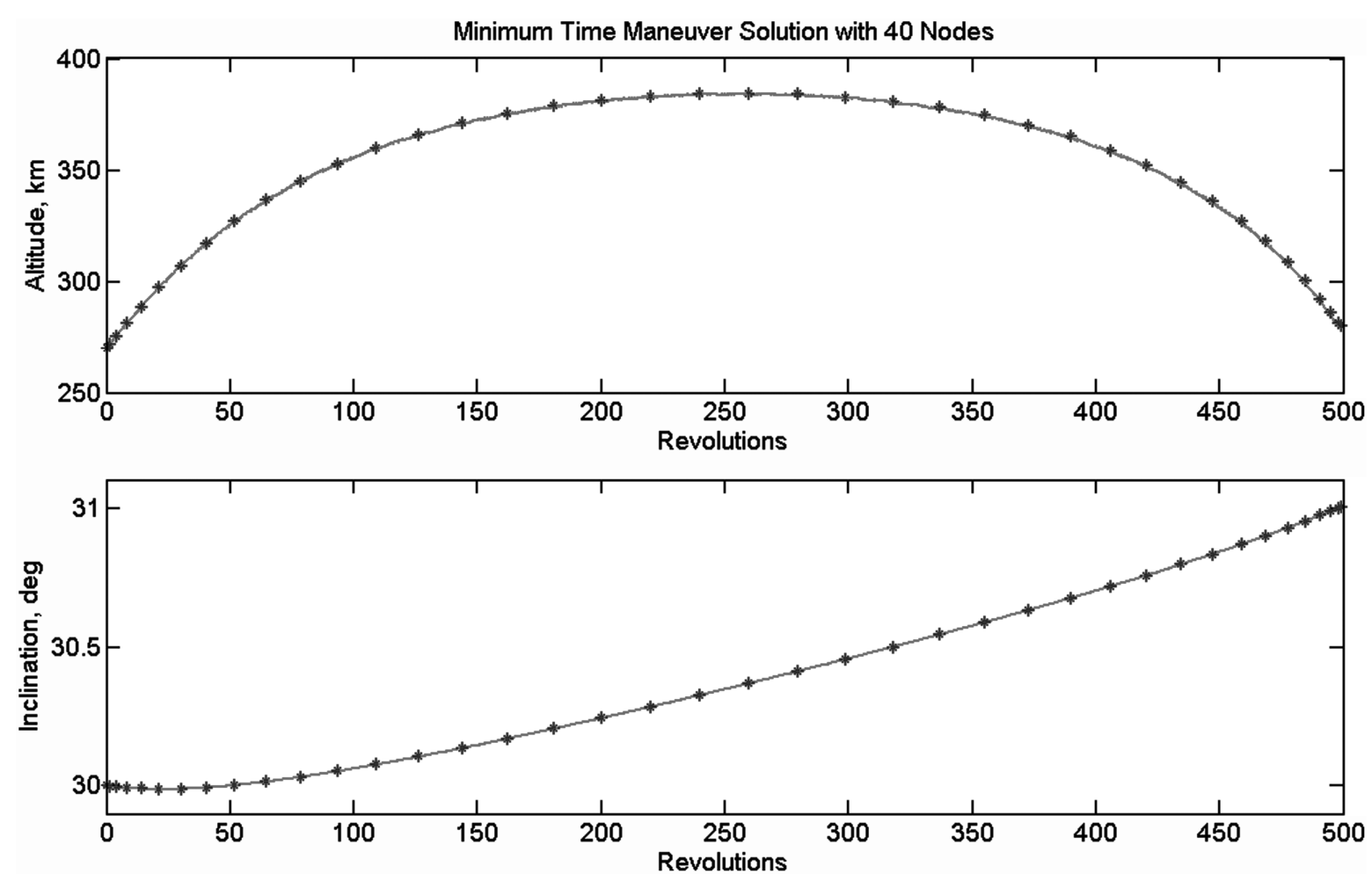

Fig. 12 Minimum time orbit change trajectory with drag.

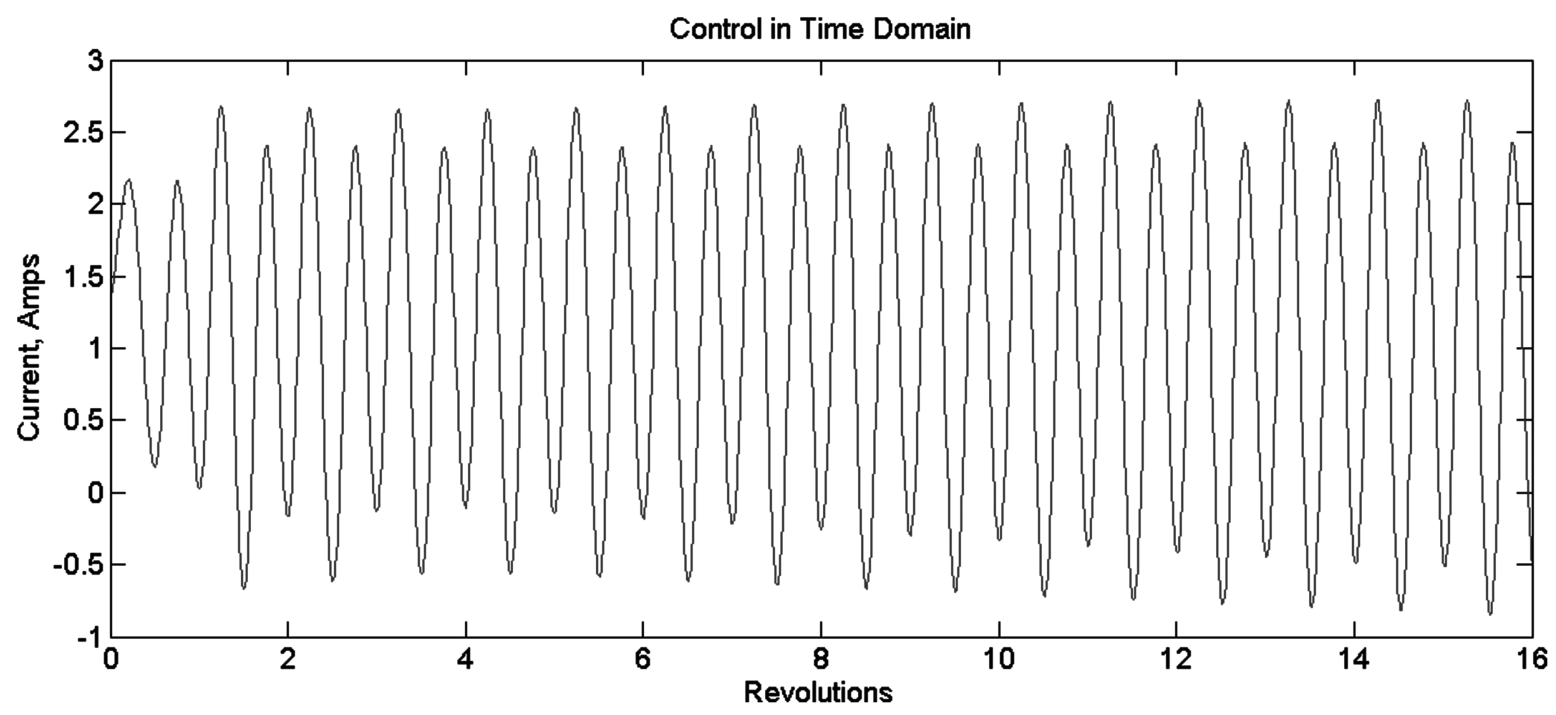

Fig. 13 Current control in time domain for the first 16 revolutions for minimum time maneuver with drag.

component of the control current is throttled (see Fig. 11) such that the satellite initially climbs and then descends to the final orbit to avoid the cost of increased drag at lower altitudes as much as possible as shown in Fig. 12. Contending with drag, this EDT takes an additional four days to complete the maneuver.

Controlling the slowly varying current Fourier coefficients over many revolutions has the advantage of solving long-term problems with relatively few nodes in the optimization algorithm. A similar problem solved using a small time scale and exact equations of motion would yield the instantaneous states during each revolution; however, it would require an exorbitant number nodes over the same time frame to arrive at a meaningful solution. The periodic current would require at least four nodes per orbit revolution in the short time scale domain to establish a control current that avoids aliasing. The first day alone in this example consists of 32 control current cycles (Fig. 13), which would require at least 64 nodes to adequately capture all the cycles. Using large time scales and averaged states, we have solved a multirevolution orbital maneuvering problem using 40 optimization nodes contrasted to the 2000 nodes that would have been required using a short time scale and instantaneous states.

\section{Conclusions}

Solving optimal control problems in Fourier space using large time scales and time-averaged orbital states has significant advantages when the desire is to control the secular behavior of a continuously operating, low-thrust satellite system over a long time rather than the instantaneous periodic behavior. In satellite control, a rapidly changing periodic variable may be averaged out, leaving only the dynamics of the slowly changing variables. In this paper, we investigated a method of constructing and solving a large time scale optimal control problem using an electrodynamic tether to maneuver 
to a desired orbit. Optimal controls for three sample maneuvers spanning up to 500 orbital revolutions were determined using 30-40 optimization nodes. This method of optimal control in Fourier space could assist engineers with initial trade studies to determine design and performance parameters for a tether or any other low-thrust maneuvering satellite. Implemented as a far horizon controller, it could determine a long-term control strategy and feed commands to another controller operating over short time scales to manage instantaneous states such as tether libration.

\section{Appendix: Derivation of Averaged Equations of Motion}

This appendix provides a derivation of secular equations of motion using a mixed set of classical and equinoctial coordinates and the method of averaging. To determine the secular change in a given state $x_{i}$, we use the approximation

$$
\mathrm{d} t \approx \frac{1}{n}(1-2 e \cos v) \mathrm{d} v
$$

and integrate over $N$ periods as follows:

$$
\Delta x_{i}=\int_{t_{0}}^{t_{f}} \mathrm{~d} x_{i} \approx \frac{1}{n} \int_{0}^{2 \pi N} \frac{\mathrm{d} x_{i}}{\mathrm{~d} t}(1-2 e \cos v) \mathrm{d} \nu
$$

Because the orbits considered are nearly circular, eccentricity is very small, and the argument of perigee is ill defined. Therefore, two equinoctial coordinates defined as $h=e \sin \omega$ and $k=e \cos \omega$ are better suited for this orbit type. Thus, changes in semimajor axis, inclination, and right ascension of the ascending node are approximated as

$$
\begin{aligned}
\Delta a & \approx 2 C a \cos i\left(\frac{1}{n}\right) \int_{0}^{2 \pi N} I(1+2 e \cos v) \mathrm{d} v \\
- & \frac{2 D}{n} \int_{0}^{2 \pi N}\left(1+2 e \cos v+\frac{a e \cos v}{h^{*}}\right)(1-2 e \cos v) \mathrm{d} v \\
\Delta i & \approx-C \sin i\left(\frac{1}{n}\right) \int_{0}^{2 \pi N} I \cos ^{2}(\omega+v) \mathrm{d} v \\
& =-C \sin i\left(\frac{1}{n}\right) \int_{0}^{2 \pi N} \frac{I}{e^{2}}\left(k^{2} \cos ^{2} v+h^{2} \sin ^{2} v-h k \sin 2 v\right) \mathrm{d} v \\
\Delta \Omega & \approx-\frac{C}{2}\left(\frac{1}{n}\right) \int_{0}^{2 \pi N} I \sin (2 v+2 \omega) \mathrm{d} v \\
& =-\frac{C}{2}\left(\frac{1}{n}\right) \int_{0}^{2 \pi N} \frac{I}{e^{2}}\left[h k \cos 2 v+\frac{1}{2}\left(k^{2}-h^{2}\right) \sin 2 v\right] \mathrm{d} v
\end{aligned}
$$

where $C=L \gamma_{m} / n m a^{4}$ represents the thrust per unit current, and the drag rate is $D=B^{*} \mu \rho(a) / 2 n a$.

The only control that will yield nonzero solutions after integrating these equations is a periodic current. The control current may be expressed as the sum of the periodic functions that produce secular changes to the states; therefore, we use the first five terms of a Fourier series [Eq. (1)]. After integration, we obtain the secular changes to three of the five states that change on a long time scale.

$$
\begin{aligned}
& \Delta a \approx\left[2 C a \cos i\left(u_{1}+u_{2} e\right)-2 D\right] \frac{2 \pi N}{n} \\
& \Delta i \approx-C \sin i\left(\frac{u_{1}}{2}+\frac{k^{2}-h^{2}}{4 e^{2}} u_{4}-\frac{h k}{2 e^{2}} u_{5}\right) \frac{2 \pi N}{n} \\
& \Delta \Omega \approx-C\left(\frac{h k}{2 e^{2}} u_{4}+\frac{k^{2}-h^{2}}{4 e^{2}} u_{5}\right) \frac{2 \pi N}{n}
\end{aligned}
$$

These results are consistent with [3].

The time derivatives of the equinoctial coordinates may be calculated as follows:

$$
\begin{aligned}
\dot{h} & =e \cos \omega \dot{\omega}+\dot{e} \sin \omega \approx C I \cos i[\cos (v+\omega)(h \cos v+k \sin v) \\
& \times(1+2 e \cos v) \cos \omega+(2-e \cos v)(1+3 e \cos v) \sin v \cos \omega \\
& \left.+\left(2 \cos v+e \sin ^{2} v\right)(1+3 e \cos v) \sin \omega\right] \\
& -\frac{2 D}{a}\left\{\frac{\sin v}{2 e}\left[1+\left(1+\frac{a}{h^{*}}\right) e \cos v\right](2-e \cos v) \cos \omega\right. \\
& \left.+\left[\cos v+e+\left(1+\frac{a}{h^{*}}\right) e \cos ^{2} v\right] \sin \omega\right\}
\end{aligned}
$$

Carrying out the multiplications, eliminating second and higher order eccentricity terms, then substituting in $h$ and $k$, we write

$$
\begin{aligned}
\dot{h} \approx & C I \cos i\left\{\left[\left(\frac{k}{e} \cos v-\frac{h}{e} \sin v+2 k \cos ^{2} v-h \sin 2 v\right)\right.\right. \\
& \left.\times(h \cos v+k \sin v)+\left(2 \sin v+\frac{5}{2} e \sin 2 v\right)\right] \frac{k}{e} \\
& \left.+\frac{2 h}{e} \cos v+h+5 h \cos ^{2} v\right\} \\
& -\frac{2 D}{a}\left\{\left[\frac{\sin v}{e}+\frac{a}{2 h^{*}} \sin 2 v-\left(1+\frac{a}{h^{*}}\right) \frac{e}{2} \cos ^{2} v \sin v\right] \frac{k}{e}\right. \\
& \left.+\left[\cos v+e+\left(1+\frac{a}{h^{*}}\right) e \cos ^{2} v\right] \frac{h}{e}\right\}
\end{aligned}
$$

recognizing that

$$
\cos (v+\omega)=\cos v \cos \omega-\sin v \sin \omega=\frac{k}{e} \cos v-\frac{h}{e} \sin v
$$

and $e^{2}=h^{2}+k^{2}$.

Integrating with respect to the true anomaly from 0 to $2 \pi N$, we find the change in the average $h$ state:

$$
\begin{aligned}
\Delta h & \approx\left\{C \operatorname { c o s } i \left[u_{1}\left(\frac{3 h}{2}\right)+u_{2}\left(\frac{h}{e}\right)+u_{3}\left(\frac{k}{e}\right)+u_{4}\left(\frac{h}{4}+\frac{h k^{2}}{2 e^{2}}\right)\right.\right. \\
& \left.\left.+u_{5}\left(\frac{k}{4}+\frac{\left(k^{2}-h^{2}\right) k}{4 e^{2}}\right)\right]-\frac{D}{a}\left(1+\frac{a}{h^{*}}\right) h\right\} \frac{2 \pi N}{n}
\end{aligned}
$$

We obtain the $k$ state dynamics in a similar manner:

$$
\begin{aligned}
\dot{k} & =-e \sin \omega \dot{\omega}+\dot{e} \cos \omega \approx C I \cos i[-\cos (v+\omega)(h \cos v+k \sin v) \\
& \times(1+2 e \cos v) \sin \omega-(2-e \cos v)(1+3 e \cos v) \sin v \sin \omega \\
& \left.+\left(2 \cos v+e \sin ^{2} v\right)(1+3 e \cos v) \cos \omega\right] \\
& -\frac{2 D}{a}\left\{\frac{-\sin v}{2 e}\left[1+\left(1+\frac{a}{h^{*}}\right) e \cos v\right](2-e \cos v) \sin \omega\right. \\
& \left.+\left[\cos v+e+\left(1+\frac{a}{h^{*}}\right) e \cos ^{2} v\right] \cos \omega\right\}
\end{aligned}
$$

Rewriting Eq. (5), using definitions of $h$ and $k$, we obtain

$$
\begin{aligned}
\dot{k} \approx & C I \cos i\left\{\left[-\left(\frac{k}{e} \cos v-\frac{h}{e} \sin v+2 k \cos ^{2} v-h \sin 2 v\right)\right.\right. \\
& \left.\times(h \cos v+k \sin v)-\left(2 \sin v+\frac{5}{2} e \sin 2 v\right)\right] \frac{h}{e} \\
& \left.+\frac{2 k}{e} \cos v+k+5 k \cos ^{2} v\right\} \\
& -\frac{2 D}{a}\left\{-\left[\frac{\sin v}{e}+\frac{a}{2 h^{*}} \sin 2 v-\left(1+\frac{a}{h^{*}}\right) \frac{e}{2} \cos ^{2} v \sin v\right] \frac{h}{e}\right. \\
& \left.+\left[\cos v+e+\left(1+\frac{a}{h^{*}}\right) e \cos ^{2} v\right] \frac{k}{e}\right\}
\end{aligned}
$$

Integrating with respect to the true anomaly from 0 to $2 \pi N$, we find the change in the $k$ state: 


$$
\begin{aligned}
\Delta k & \approx\left\{C \operatorname { c o s } i \left[u_{1}\left(\frac{3 k}{2}\right)+u_{2}\left(\frac{k}{e}\right)+u_{3}\left(\frac{-h}{e}\right)+u_{4}\left(\frac{k}{4}-\frac{h^{2} k}{2 e^{2}}\right)\right.\right. \\
& \left.\left.+u_{5}\left(-\frac{h}{4}+\frac{\left(h^{2}-k^{2}\right) h}{4 e^{2}}\right)\right]-\frac{D}{a}\left(1+\frac{a}{h^{*}}\right) k\right\} \frac{2 \pi N}{n}
\end{aligned}
$$

\section{Acknowledgments}

R. Stevens thanks Mike Ross for his assistance in efficiently transforming the math problem into a numerical one that is well conditioned for numerical optimization. R. Stevens also thanks Joe Carroll for providing insights into tether design and controls that helped form the basis for using long time scales in optimal control problems.

\section{References}

[1] Williams, P., "Optimal Orbital Transfer with Electrodynamic Tether," Journal of Guidance, Control, and Dynamics, Vol. 28, No. 2, 2005, pp. 369-371. doi: $10.2514 / 1.12016$

[2] Carroll, J., "Guidebook for Analysis of Tether Applications," Report for Martin Marietta Corp, Contract, RH4-394049, March 1985, p. 31.
[3] Tragesser, S. G., and San, H., "Orbital Maneuvering with Electrodynamic Tethers," Journal of Guidance, Control, and Dynamics, Vol. 26, No. 5, 2003, pp. 805-810.

[4] Ross, I. M., "A Beginner's Guide to DIDO," Ver. 7.3, Document \# TR710, Elissar, LLC, Monterey, CA, 2007.

[5] DIDO, A Matlab® Application Software Package for Solving Optimal Control Problems, Ver. 2003b, Elissar, Monterey, CA, 2003.

[6] Wiesel, W., Modern Astrodynamics, Aphelion Press, Beavercreek, OH, 2003, pp. 147-151.

[7] Meirovitch, L., Methods of Analytical Dynamics, Dover, New York, 1998, pp. 322-325.

[8] Levin, E., Dynamic Analysis of Space Tether Missions, Advances in the Astronautical Sciences, Vol. 126, American Astronomical Society Publication, San Diego, CA, 1993, pp. 115-116.

[9] Ross, I. M., and Fahroo, F., Legendre Pseudospectral Approximations of Optimal Control Problems, Vol. 295,Lecture Notes in Control and Information Sciences, Springer-Verlag, New York, 2003, pp. 327-342.

[10] Ross, I. M., Gong, Q., and Sekhavat, P., "Low-Thrust, High-Accuracy Trajectory Optimization," Journal of Guidance, Control, and Dynamics, Vol. 30, No. 4, 2007, p. 921-933. doi:10.2514/1.23181

[11] Gong, Q., Ross, I. M., Kang, W., and Fahroo, F., "On the Pseudospectral Covector Mapping Theorem for Nonlinear Optimal Control," 45th IEEE Conference on Decision and Control, IEEE, San Diego, CA, Dec. 2006, pp. 2679-2686. 